\title{
Efficacy and safety of programmed death I inhibitors in patients with advanced non-small cell lung cancer: a meta-analysis
}

This article was published in the following Dove Press journal:

Cancer Management and Research

Yi Liu',*

Sijing Zhou ${ }^{2, *}$

Yongsheng $\mathrm{Du}^{3, *}$

Li Sun'

Huihui Jiang'

Binbin Zhang'

Gengyun Sun'

Ran Wang

'Department of Respiratory and Critical Care Medicine, The First Affiliated Hospital of Anhui Medical University, Hefei, 230022, People's Republic of China; ${ }^{2}$ Hefei Prevention and Treatment Center for Occupational Diseases, Hefei 230022, People's Republic of China; ${ }^{3}$ Department of General Medicine, Hefei Second People's Hospital, Hefei 230022, People's Republic of China

*These authors contributed equally to this work
Correspondence: Ran Wang; Gengyun Sun Department of Respiratory and Critical Care Medicine, The First Affiliated Hospital of Anhui Medical University, Hefei 230022, People's Republic of China

Tel +86 55I $62922914 ;+8655162922913$ Fax +8655I 6292 2914; +8655I 62922913 Email ranwangtjmu@hotmail.com; sungengyun@hotmail.com
Objective: This study aims to perform systematic review and meta-analysis of all randomized controlled trials that compare the efficacy and safety of programmed death 1 (PD-1) inhibitors versus chemotherapy alone in previously untreated advanced non-small cell lung cancer (NSCLC). Materials and methods: Several databases, including Medline, Cochrane Library, Embase, and Web of Science, were searched. The main outcome measures included overall survival (OS), progression-free survival (PFS), objective response rate (ORR), and adverse events (AEs).

Results: The results of meta-analysis are expressed as the hazard ratio (HR) or risk ratio (RR) with their corresponding 95\% confidence intervals (CIs). The final analysis included six trials for 3,930 patients. PD-1 inhibitors led to a statistically superior survival benefit over chemotherapy in patients with advanced NSCLC. OS was longer in patients who received PD-1 inhibitors $(\mathrm{HR}=0.71,95 \% \mathrm{CI}=0.62-0.74, P=0.000)$. Furthermore, PD- 1 inhibitors had significantly higher objective response rate than chemotherapy $(\mathrm{RR}=0.20,95 \% \mathrm{CI}$ $=0.17-0.23, P=0.000)$. Meta-analysis showed that the AEs of any grade with PD- 1 inhibitors were lower than those with chemotherapy $(\mathrm{RR}=0.78 ; 95 \% \mathrm{CI}=0.75-0.81, P=0.000)$.

Conclusion: PD-1 inhibitors showed a clinically meaningful survival benefit and an improved safety profile in patients with previously treated NSCLC.

Keywords: programmed death 1, lung cancer, efficacy, safety

\section{Introduction}

The management of non-small cell lung cancer (NSCLC) has evolved enormously. Multiple treatment modalities, including surgery, chemotherapy, targeted therapy, and radiotherapy, all led to robust improvements in the outcomes of this disease. ${ }^{1}$

Cytotoxic chemotherapy has become the backbone of treatment for patients with advanced NSCLC for decades. Standard platinum-based doublets in unselected patient populations have resulted in a median survival of 8-10 months. ${ }^{2}$ With the advent of targeted therapy, new therapeutic models have focused on targeting the immune system. Programmed death 1 (PD-1) is an immune checkpoint receptor that is expressed on activated $\mathrm{T}$ cells and hinders the immune response to protect against excessive inflammation and the development of autoimmunity. ${ }^{3}$

PD-1 is a member of the immunoglobulin B7-CD28 family and can be expressed on activated T cells. ${ }^{4}$ This receptor is often considered as a marker of T cell exhaustion. Effector T-cell function is inhibited when engaged by its ligand, PD-L1, which can be expressed on both tumor and immune cells. PD-L1 is the major ligand for PD-1 and is overexpressed in many malignant tumor cells, including NSCLC. ${ }^{3,5,6}$ In patients with 
cancer, the expression of PD-1 on tumor-infiltrating lymphocytes and its interaction with the ligands on tumor and immune cells in the tumor microenvironment undermine antitumor immunity and support its rationale for PD-1 blockade in cancer immunotherapy. ${ }^{7}$ Tumor cell expression of PDL1 is a mechanism of tumor escape from a specific T-cell response. $^{6}$ The combination of PD-1 and PD-L1 can inhibit the proliferation and viability of CD4+ T cells and CD8+ $\mathrm{T}$ cells, thereby reducing the immune response to the surrounding tissues and preventing autoimmune diseases in humans. ${ }^{8}$ One hallmark of cancer is immune evasion in which the immune system does not mount an effective antitumor response. ${ }^{9}$ The combination of PD-1 and PD-L1 in patients with tumor can reduce the $\mathrm{T}$ cell immune killing effect of tumor local microenvironment, leading to tumor immune escape and tumor growth stimulation. ${ }^{10}$

Blocking the PD-1 pathway can theoretically restore the immune killing effect of T cells in the tumor microenvironment and successfully inhibit tumors. Two antibodies against PD-1 (nivolumab and pembrolizumab) have been approved by the US Food and Drug Administration (FDA) for treating patients with NSCLC and other tumor types. ${ }^{11}$ Several clinical trials are currently evaluating an expansion of the indication for PD-1 inhibitions as monotherapy or in combination with other treatments. Herein, we performed a meta-analysis of randomized controlled trials (RCTs) and summarized evidence of the efficacy and safety of PD-1 inhibitor monotherapy in patients with advanced NSCLC. The completed RCT showed that PD-1 inhibitors remarkably prolonged the survival benefits of patients with NSCLC.

\section{Materials and methods}

This meta-analysis was conducted in accordance with the PRISMA guidelines. The studies included in this metaanalysis are previously published, and therefore, ethical approval and informed consent are not required.

\section{Inclusion and exclusion criteria}

Only the RCTs that evaluated the effectiveness or safety of PD-1 inhibitor monotherapy in patients with NSCLC were eligible. In addition, patients had to be diagnosed with NSCLC by pathological examination, and only unresectable NSCLC such as stage IIIB, stage IV, and so on, was considered. Patients previously treated with chemotherapy and surgical operation were acceptable, but those who had previously received immune checkpoint inhibitor therapy were excluded. Considering an increased risk of pneumonitis, patients who received more than 30 Gy of radiotherapy to the lung in the previous 6 months were also excluded. Additionally, phase I and single-arm phase II trials were excluded due to their lack of control groups. No limitation was imposed on patient age, sex, or race. Trials with uncertain or marked inequality of characteristics between groups at baseline were also excluded. Two investigators reviewed the articles independently to exclude irrelevant and overlapping studies. The results were compared, and disagreements were resolved by discussion and consensus. The process of inclusion and exclusion of the literature is presented in Figure 1.

\section{Search strategy and study selection}

We searched electronic databases for literature published up to August 2018; these databases were Medline, Embase, Cochrane Library, and Web of Science. We used advanced search for all databases.

The search terms used were ("Opdivo" [All Fields]), ("Nivolumab" [All Fields]), ("Pembrolizumab" [All Fields]), ("Keytruda" [All Fields]), ("PD-1” [All Fields]), ("PD-1 antibody" [All Fields]), ("PD-1 inhibitors" [All Fields]), ("immune checkpoint" [All Fields]), ("MK3475" [All Fields]), or ("BMS - 936558" [All Fields]) and ("non-small cell lung cancer" [All Fields]), ("NSCLC" [All Fields]), ("lung cancer" [All Fields]), or ("non-small-cell lung carcinoma" [All Fields]).

\section{Efficacy indicators}

The main outcome measures included overall survival (OS), progression-free survival (PFS), overall response rate (ORR), adverse events (AEs), and severe adverse events (SAEs) (defined as grade 3 or higher). OS refers to the time from random assignment to death, irrespective of the cause of death. For patients with no event observed, the time to censor refers to the time of last follow-up. PFS denotes the time from randomization to the first occurrence of disease progression. One or more outcome measures had to be extracted from a trial for it to be included in the meta-analysis.

The treatment efficacy of PD-1 inhibitors versus chemotherapy was measured by a hazard ratio (HR) for OS and PFS, and a risk ratio (RR) for AEs, objective response rate (ORR), and SAEs, with their corresponding 95\% confidence intervals (CIs).

\section{Data extraction}

Two investigators searched the publications independently by using standardized data abstraction forms. The name of 


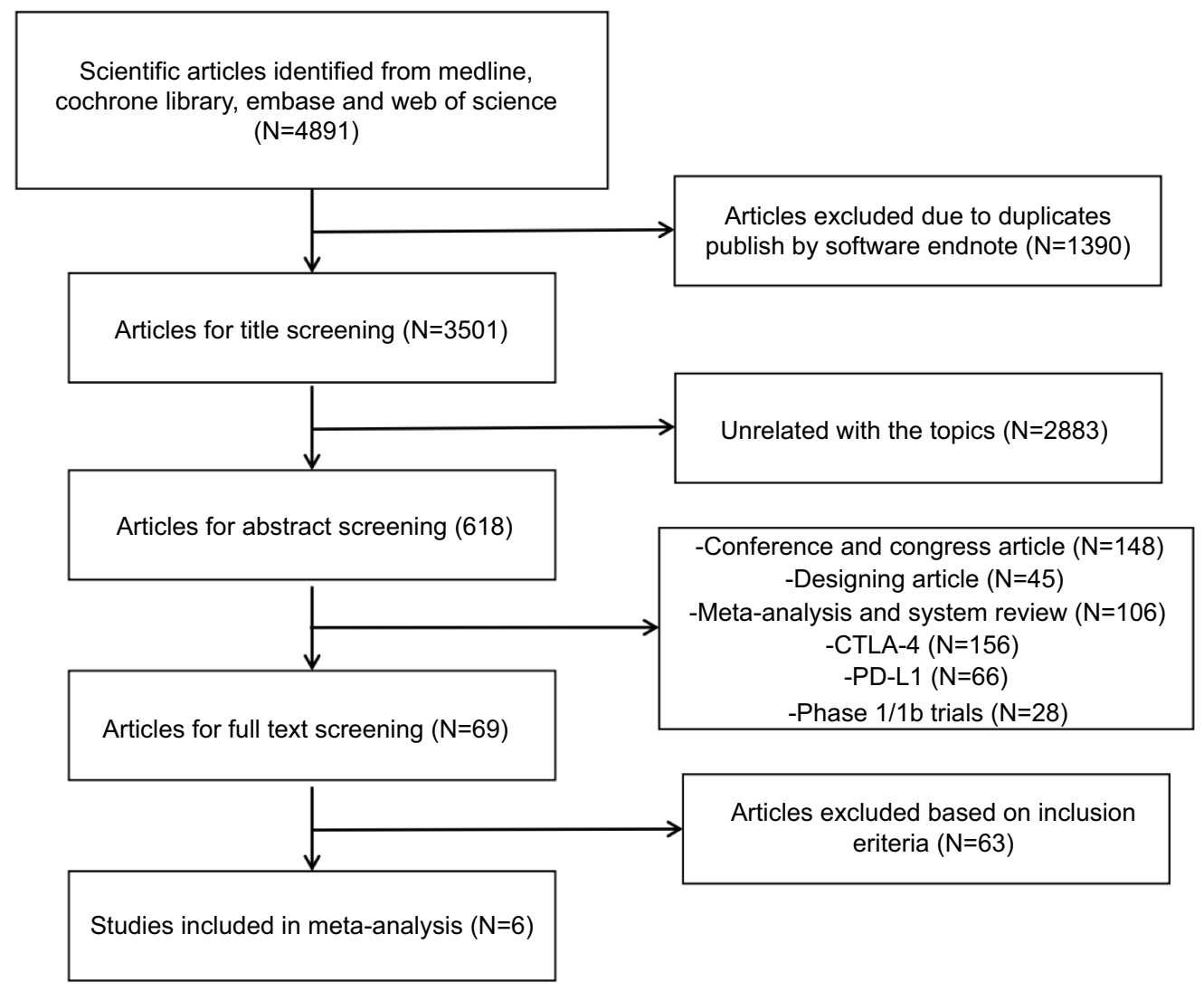

Figure I Flow diagram of the literature search and trial selection process.

the first author and the year of publication were used to identify the study. From each study, the following information were extracted: first author's surname, year of publication, trial phase, number of subjects, interventions, histology, and outcomes (OS, PFS, AEs, ORR, and SAEs) (Table 1).

\section{Quality assessment}

The Cochrane Collaboration's tool was utilized to assess the risk of RCT bias, considering the following aspects: whether the method of allocation was truly random, whether proper concealment of allocation existed, whether equality occurred between the two groups at baseline in terms of prognostic features, whether the eligibility criteria were described, whether blinding of the outcome assessors was performed, whether loss to follow-up in each treatment arm was demonstrated, and whether intention-totreat (ITT) analysis was considered (Table 2). ${ }^{12}$

\section{Statistical analysis}

All analyses were performed using Stata 12.0. The results of the meta-analysis were expressed as the hazard ratio (HR) or risk ratio (RR), with their corresponding 95\% CI. PFS and OS were measured in terms of HR and their corresponding 95\% CI. Risk ratio (RR) and corresponding 95\% CIs were the summary measurement of ORR and risk of AEs. (We first tested the heterogeneity between the studies using $I^{2}$ statistics). $I^{2}<50 \%$ was considered lowlevel heterogeneity, and $I^{2}>50 \%$ pertained to high-level heterogeneity. If heterogeneities existed, one of the following techniques was used to attempt to explain such heterogeneities: (1) random effect model for meta-analysis, (2) subgroup analyses, (3) sensitivity analyses. Basing on the statistical significance of the heterogeneity test, we applied a random effects model $\left(I^{2}<50 \%\right)$ or fixed effects model $\left(I^{2}>50 \%\right)$ to calculate the pooled results. Descriptive analysis was used if the heterogeneity between the groups was excessively large. $P<0.05$ was considered statistically significant.

\section{Results}

\section{Articles included}

The initial search yielded 4,891 records from Medline, Cochrane Library, Embase, and Web of Science. Of these, 1,390 studies were removed as duplicate records, and 69 potential studies were identified for the full-text 


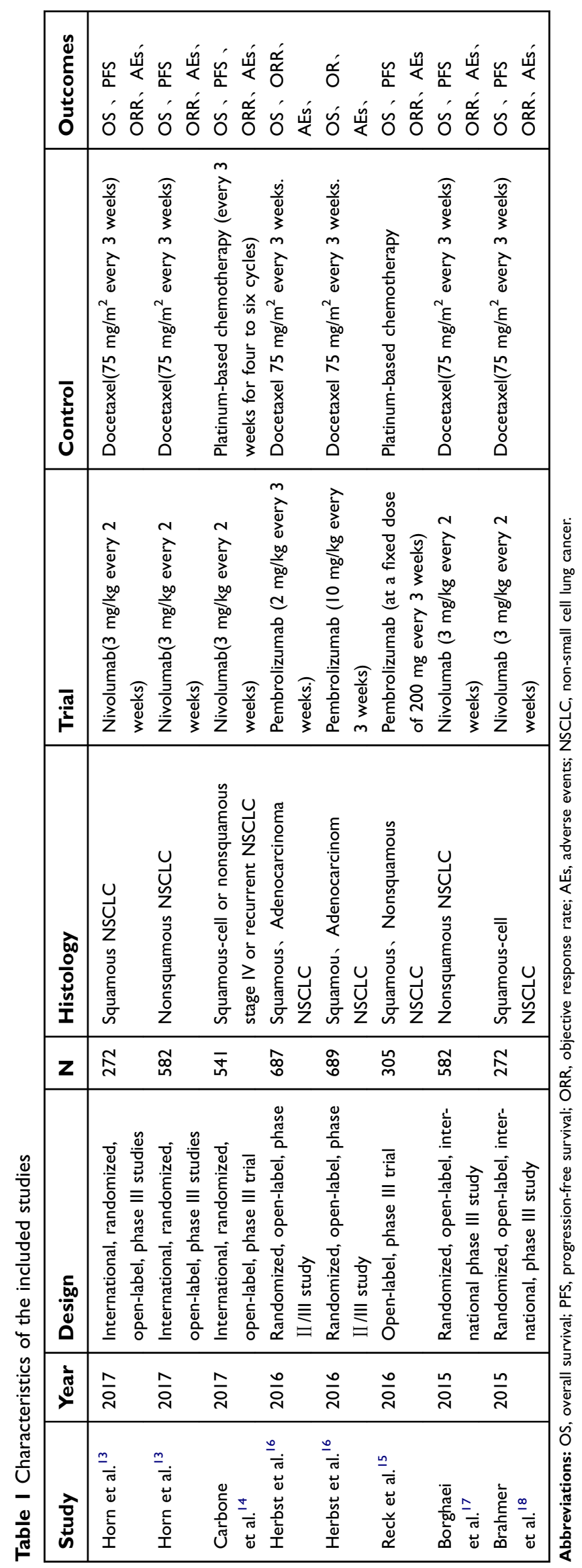


Table 2 Quality assessment by the Cochrane Collaboration's tool

\begin{tabular}{|c|c|c|c|c|c|c|c|}
\hline Reference & $\begin{array}{l}\text { Random } \\
\text { Sequence } \\
\text { generation }\end{array}$ & $\begin{array}{l}\text { Allocation } \\
\text { concealment }\end{array}$ & $\begin{array}{l}\text { Blinding of } \\
\text { participants } \\
\text { and } \\
\text { personnel }\end{array}$ & $\begin{array}{l}\text { Blinding of } \\
\text { outcome } \\
\text { assessment }\end{array}$ & $\begin{array}{l}\text { Incomplete } \\
\text { outcome } \\
\text { data }\end{array}$ & $\begin{array}{l}\text { Selective } \\
\text { reporting }\end{array}$ & $\begin{array}{l}\text { Other } \\
\text { bias }\end{array}$ \\
\hline Horn, L. $2017^{13}$ & High risk & Unclear risk & Unclear risk & Unclear risk & Unclear risk & Unclear risk & Unclear risk \\
\hline Carbone, D.P. $2017^{14}$ & Unclear risk & Unclear risk & Unclear risk & Low risk & Low risk & Unclear risk & Unclear risk \\
\hline Reck, M. $2016^{15}$ & Low risk & Unclear risk & Unclear risk & Low risk & Low risk & Unclear risk & Unclear risk \\
\hline Herbst, R. S. $2016^{16}$ & Unclear risk & Unclear risk & High risk & High risk & High risk & Unclear risk & Unclear risk \\
\hline Borghaei, H. $2015^{17}$ & Unclear risk & Unclear risk & High risk & High risk & High risk & Unclear risk & Unclear risk \\
\hline Brahmer, J. $2015^{18}$ & Unclear risk & Unclear risk & High risk & High risk & High risk & Unclear risk & Unclear risk \\
\hline
\end{tabular}

review. Among them, phase I and single-arm phase II trials were excluded due to their lack of control groups. Six RCTs (involving 3,930 patients) met the inclusion criteria, and they were included in this meta-analysis. Figure 1 outlines the selection process in detail.

\section{Effectiveness}

\section{OS and median OS $(95 \% \mathrm{Cl})$}

All of the studies reported OS and Median OS (95\% CI) data. The median OS (95\% CI) in the PD-1 inhibitor monotherapy and chemotherapy groups was 14.4 (95\% CI, 11.7-17.4) and 13.2 months (95\% CI, 10.7-17.1) in Carbone (2017), ${ }^{14} 9.2$ (95\% CI, 7.3-12.6) and 6.0 months (95\% CI, 5.1-7.3) in Horn (2017) ${ }^{13}$ arm1, 12.2 (95\% CI, 9.7-15.1) and 9.5 months (95\% CI, 8.1-10.7) in Horn (2017) arm2, 10.4 (95\% CI, 9.4-11.9) and 8.5 months (95\% CI, 7.5-9.8) in Herbst $(2016)^{16}$ arm1, 12.7 (95\% CI, 10.3-17.3) and 8.5 months (95\% CI, 7.5-9.8) in Herbst (2016) arm2, 9.2 (95\% CI, 7.3-13.3) and 6.0 months (95\% CI, 5.1-7.3) in Brahmer (2015),18 12.2 months (95\% CI, 9.7-15.0) and 9.4 months (95\% CI, 8.1-10.7) in Borghaei (2015),17 respectively. These results indicated that $\mathrm{PD}-1$ inhibitor monotherapy prolonged the survival time of patients with NSCLC.

OS was significantly longer in patients treated with PD-1 inhibitor monotherapy than with chemotherapy, with an estimated HR of $0.70(95 \% \mathrm{CI}=0.62-0.80$, test of $\mathrm{ES}=1: Z=5.63, P=0.000)$. Moreover, $52.2 \%$ heterogeneity was identified among the included studies $(P=0.041$, $I^{2}=52.2 \%$ ). We conducted a sensitivity analysis to explore the potential source of heterogeneity. The exclusion of one study conducted by Carbone (2017) yielded evident change $(\mathrm{HR}=0.68,95 \% \mathrm{CI}=0.62-0.74$, test of $\mathrm{ES}=1$ : $Z=8.75, P=0.000$; Figure 2). Furthermore, the test for heterogeneity has disappeared $\left(I^{2}=0\right)$.

\section{PFS and median PFS $(95 \% \mathrm{Cl})$}

Five studies presented data for PFS and median PFS (95\% CI). The PFS in the PD-1 inhibitor monotherapy and chemotherapy groups was 4.2 (95\% CI, 3.0-5.6) and 5.9 months (95\% CI, 5.4-6.9) in Carbone (2017), 3.9 (95\% CI, 3.1-4.1) and 4 months (95\% CI, 3.1-4.2) in Herbst (2016) arm1, 4 (95\% CI, 2.7-4.3) and 4 months (95\% CI, 3.1-4.2) in Herbst (2016) arm2, 3.5 (95\% CI, 2.1-4.9) and 2.8 months (95\% CI, 2.1-3.5) in Brahmer (2015), 2.3 (95\% CI, 2.2-3.3) and 4.2 months (95\% CI, 3.5-4.9) in Borghaei (2015), respectively.

The combined estimates indicated that PD-1 inhibitor monotherapy may be related to PFS (HR $=0.82,95 \%$ $\mathrm{CI}=0.69-0.96$, test of $\mathrm{ES}=1: Z=2.30, P=0.022$ ). The difference was statistically significant. However, the test for heterogeneity was significant $\left(P=0.000, I^{2}=79.6 \%\right)$. Therefore, we conducted the sensitivity analysis to explore the potential source of heterogeneity. The exclusion of one study conducted by Reck (2016) ${ }^{15}$ yielded similar results (HR $=0.87,95 \% \mathrm{CI}=0.76-1.00$, test of $\mathrm{ES}=1: Z=2.17$, $P=0.030)$. The test for heterogeneity was still significant $\left(I^{2}=85.3 \%\right)$. When we excluded the five other studies individually, the overall estimates and heterogeneity did not change substantially. Other attempts to diminish the heterogeneity by excluding studies based on clinical characteristics also failed. The results illustrated that PD-1 inhibitor monotherapy did not prolong the PFS in patients with NSCLC when used with nivolumab (HR $=0.89,95 \%$ CI $=0.72-1.10, I^{2}=82.6 \%$, test of $\mathrm{ES}=1: Z=0.82$, $P=0.411) \quad$ or pembrolizumab $\quad(\mathrm{HR}=0.72,95 \% \quad \mathrm{CI}$ $=0.55-0.95, I^{2}=80.1 \%$, test of $\mathrm{ES}=1: Z=2.33, P=0.020$; Figure 3).

The pooled results indicated that patients with NSCLC who were treated with PD-1 inhibitor monotherapy had similar PFS with those treated with chemotherapy alone. Compared 
Study

ID
$\%$

OS-HR $(95 \% \mathrm{Cl})$ weight

$0.62(0.47,0.80) 10.81$

$0.75(0.63,0.91) 22.61$

$0.71(0.58,0.88) 17.59$

$0.61(0.49,0.75) 16.88$

$0.60(0.41,0.89) 5.09$

$0.59(0.44,0.79) 8.93$

$0.73(0.59,0.89) 18.09$

$0.68(0.62,0.74) 100.00$

NOTE: Weights are from random effects anạlysis

$.41 \quad .68$

Figure 2 Forest plots of overall survival for PD-I inhibitors monotherapy or chemotherapy.

Abbreviations: OS, overall survival; HR, hazard ratio.

Study

ID
$\%$

PFS-HR $(95 \% \mathrm{Cl})$ Weight

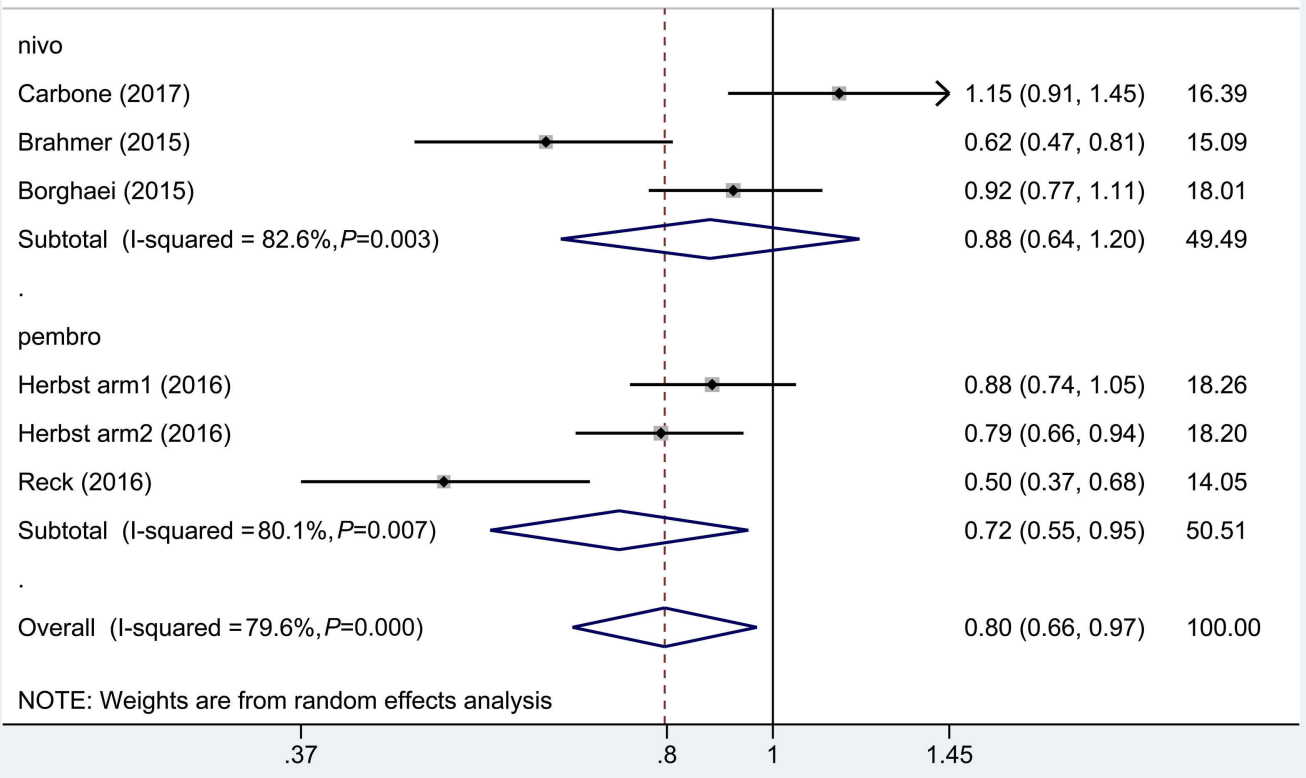

Figure 3 Subgroup hazard ratio of progression-free survival for PD-I inhibitors monotherapy or chemotherapy. Abbreviations: PFS, progressive-free survival; HR, hazard ratio. 
with chemotherapy, PD-1 inhibitor monotherapy did not improve the PFS.

\section{Objective response rate}

Five studies reported objective response rate (ORR) data. The objective response rate, according to Reck (2016), was $44.8 \%$ (95\% CI, 36.8-53.0) in the pembrolizumab group and $27.8 \%$ (95\% CI, 20.8-35.7) in the chemotherapy group. The confirmed objective response rate was significantly higher with nivolumab than with docetaxel (19\% [95\% CI, 15-24] vs $12 \%$ [95\%CI, 9-17], $P=0.02$ ) in Borghaei (2015). The confirmed objective response rate was significantly higher with nivolumab than with docetaxel (20\% [95\% CI, 14-28] vs 9\% [95\% CI, 5-15]; $P=0.008$ ) in Brahmer (2015).

The pooled results showed that PD-1 inhibitors significantly improved the objective response rate $(\mathrm{RR}=0.23,95 \%$ $\mathrm{CI}=0.18-0.28$, test of $\mathrm{ES}=0: Z=7.65, P=0.000$ ). Furthermore, $88.3 \%$ heterogeneity was identified among the included studies, and it was unacceptable. Therefore, we conducted a sensitivity analysis to explore the potential source of heterogeneity. When we excluded the results of Reck (2016) individually, the overall estimates and heterogeneity changed significantly $\left(I^{2}=38.8 \%, P=0.163\right)$, suggesting the source of heterogeneity. The combined estimates indicated that the combined use of PD-1 inhibitors might improve an objective response rate $\left(\mathrm{RR}=0.20,95 \% \mathrm{CI}=0.17-0.23, I^{2}=38.8 \%\right.$; test of $\mathrm{ES}=0: Z=14.33, P=0.000$ ) (Figure 4).

\section{PD-LI expression levels and overall survival (OS)}

Only two studies reported tumor PD-L1 expression levels in $1 \%$ and $10 \%$ respectively. To sum up, the rates of overall survival in the PD-L1 expression levels favored nivolumab (Figure 5). The efficacy of nivolumab, including a survival benefit, was observed regardless of tumor PD-L1 expression levels, with results showing that PD-L1 expression was neither prognostic nor predictive of efficacy in the population of patients with squamous-cell NSCLC. We think that the lack of an association between PD-L1 expression and efficacy is probably not related to the performance of the PD-L1 assay but is rather a function of complex interactions between tumors and the immune system.

\section{Safety \\ AEs}

Five studies presented data for AEs. Treatment-related AEs of any grade have been reported in patients with cancer treated with PD-1 inhibitors. The AEs of PD-1 inhibitors are different from those of traditional cytotoxic

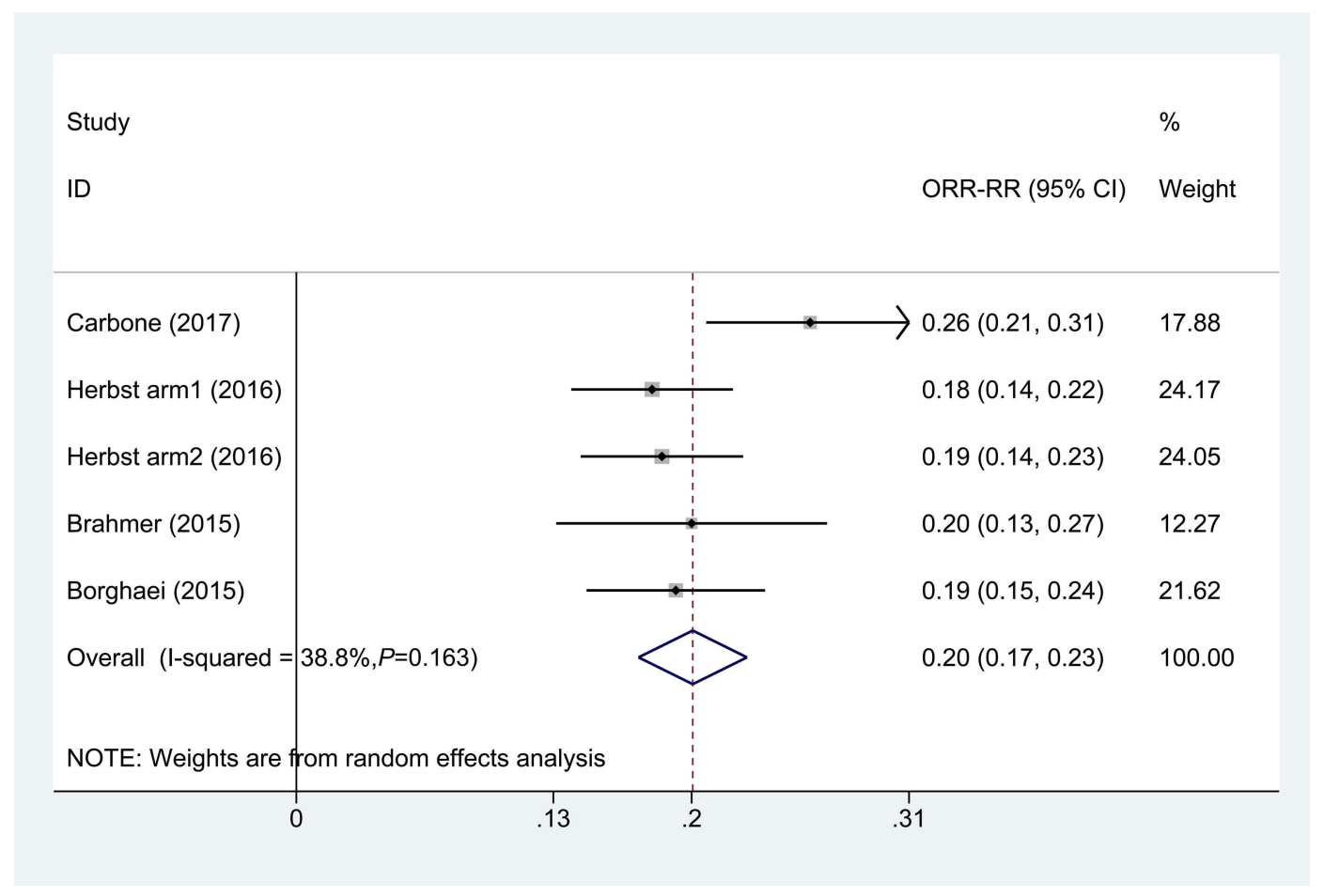

Figure 4 Forest plots of objective response rate for PD-I inhibitors monotherapy or chemotherapy Abbreviations: ORR, objective response rate; $R R$, risk ratio. 


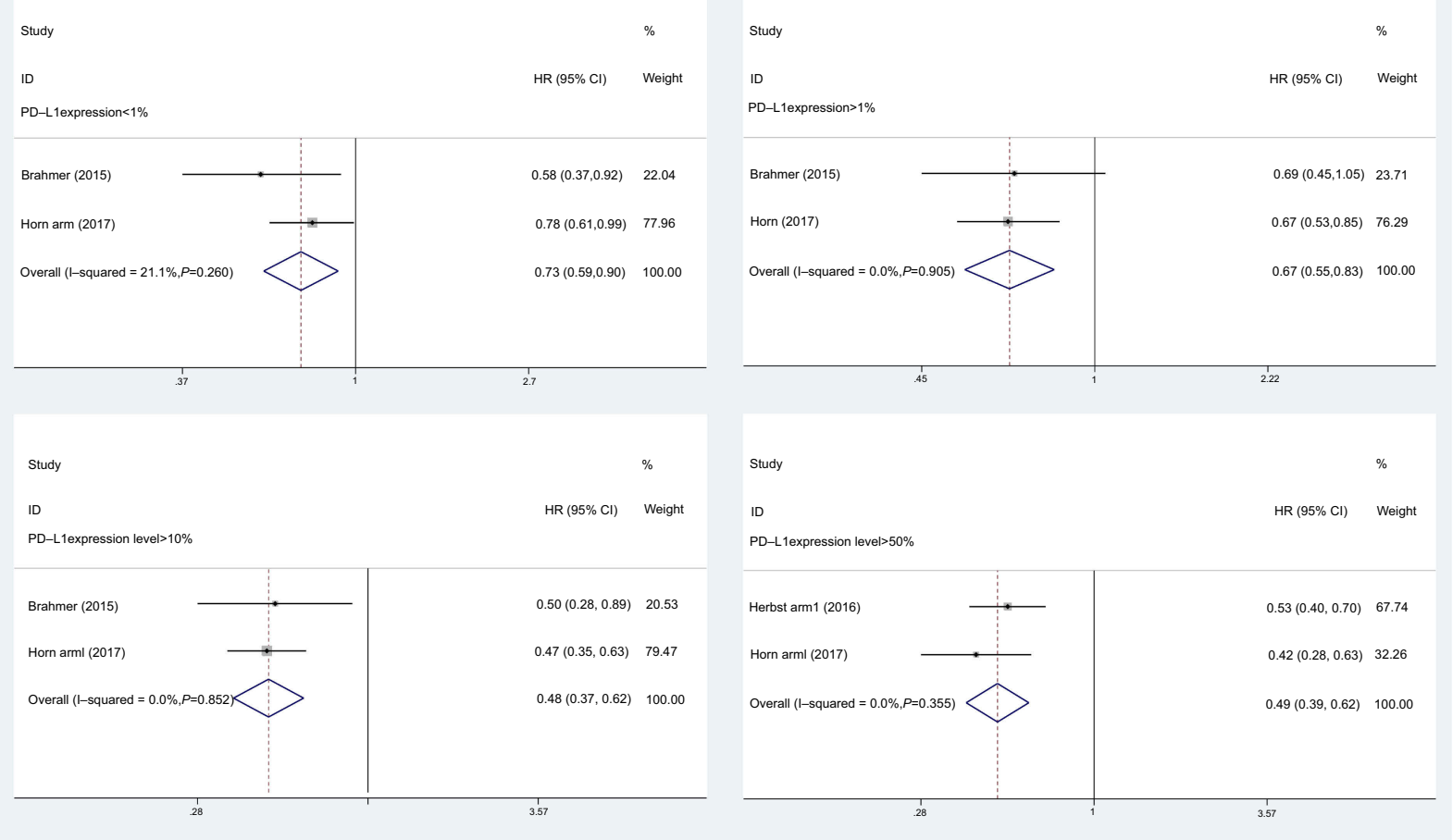

Figure 5 Forest plots of overall survival (OS) for PD-LI expression level.

Abbreviation: HR, hazard ratio.

chemotherapy to some extent. The present meta-analysis included 1,521 patients with NSCLC who received PD-1 inhibitors and 1,428 patients who received platinum-based therapy, showing a significantly increased risk of treatment-related AEs in patients with chemotherapy in the pooled analysis. The low-level heterogeneity between the studies and a fixed effect model was used to analyze the results $\left(P=0.427, I^{2}=0.0 \%\right)$. Meta-analysis showed that AEs of any grade with PD-1 inhibitors were lower than those with docetaxel for chemotherapy. The difference was statistically significant $(\mathrm{RR}=0.78,95 \% \mathrm{CI}=0.75-0.81$, $Z=11.89, P=0.000$; Figure 6 ). The safety profile of PD-1 inhibitors observed in this meta-analysis was favorable compared with that of chemotherapy.

\section{SAEs}

Five studies reported data for AEs of grade 3 or 4 . The treatment-related AEs of grade 3 or 4 with docetaxel obtained a higher rate than with nivolumab, mainly attributing to anemia and fatigue. Statistical heterogeneity existed among the studies $\left(P<0.0001, I^{2}=83.1 \%\right)$, which were analyzed using a random effects model. The results of the analysis showed that PD-1 inhibitors were significantly lower than the docetaxel-based chemotherapies in AEs of grade 3 or 4 . The difference was statistically significant $(\mathrm{RR}=0.31 ; 95 \% \mathrm{CI}=0.22,0.44 ; Z=6.72$; $P=0.000$ ). (Figure 7A)

We performed a subgroup analysis based on two antibodies against PD-1 inhibitors (nivolumab and pembrolizumab). The aggregated results illustrated that AEs of grade 3 or 4 with PD-1 inhibitors were lower than those with docetaxel for chemotherapy, when used with pembrolizumab $\left(\mathrm{RR}=0.44 ; 95 \% \mathrm{CI}=0.36,0.53 ; I^{2}=12.7 \%\right.$; $Z=8.73 ; P=0.000)$ or nivolumab $(\mathrm{RR}=0.21 ; 95 \% \mathrm{CI}$ $=0.12,0.38 ; I^{2}=83.1 ; Z=5.35 ; P=0.000$ ) (Figure 7B).

\section{Discussion}

This study reports the results of a meta-analysis that showed superior survival and an improved safety profile with PD-1 inhibitors versus the standard chemotherapy in patients with advanced and previously treated NSCLC. Herein, the OS with PD-1 inhibitor monotherapy was longer than that with chemotherapy ( $\mathrm{HR}=0.68 ; 95 \% \mathrm{CI}$ $=0.62,0.74)$. This advantage persisted with extended follow-up. However, in the primary efficacy population with stage IV or recurrent NSCLC participated in this trial, those who received first-line monotherapy with PD-1 inhibitors failed to have longer PFS than those who received chemotherapy. 


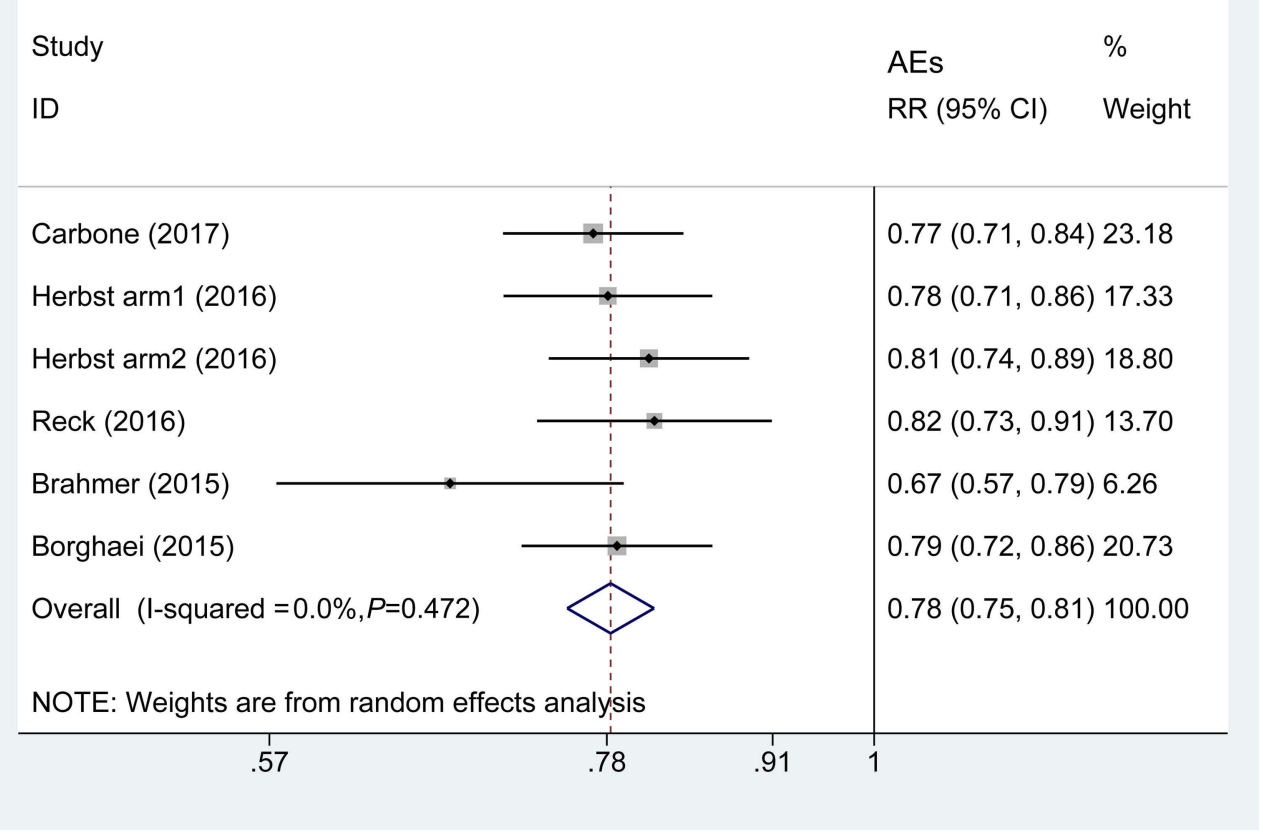

Figure 6 Forest plots of relative risks of treatment-related adverse events associated with PDI versus chemotherapy. Abbreviations: $A E s$, adverse events; RR, risk ratio.

The benefit of PD-1 inhibitors was further reflected by a significantly higher objective response rate compared with chemotherapy, and a markedly better durability of response. The duration of response with PD-1 inhibitors was longer than that with other treatment options for patients who have had disease progression during or after prior platinum-based doublet chemotherapy or targeted agents. PD-1 inhibitors were associated with significantly longer OS and a significantly higher response rate than chemotherapy but not with longer PFS. The observed results regarding PFS may be driven by subgroups of patients, as suggested by the subgroup analyses for smoking status and EGFR mutations or ALK translocations. ${ }^{19,20}$

In clinical setting, positive correlation between response to PD-1 inhibitors and mutational load, smoking signature, DNA repair pathways, and higher neoantigen burden has been observed (Rizvi et al, 2015), and more recently the association of clinical benefit form PD-1 inhibitors and higher tumor mutational burden has been confirmed. $^{21}$

Moreover, we also extract the associated information about HR of smoking status in Reck2016 (current HR 0.68 [0.36,1.31]; former HR 0.47 [0.33,0.67]; never HR 0.90 $[0.11,7.59]$ ) and Borghaei 2015 (current or former HR 0.70 $[0.56,0.86]$; never HR $1.02[0.64,1.61])$. The results approximately proved that Nivolumab would have more benefits in Smoking people.

In addition, we conducted a meta-analysis according to the PD-L1 expression levels. We found that the efficacy of nivolumab, including a survival benefit, was observed regardless of tumor PD-L1 expression levels, with results showing that PD-L1 expression was neither prognostic nor predictive of efficacy in the population of patients with squamous-cell NSCLC. We think that the lack of an association between PD-L1 expression and efficacy is probably not related to the performance of the PD-L1 assay but is rather a function of complex interactions between tumors and the immune system. Limitations of these analyses were that PD-L1 expression was assessed in archival tumor tissue, which may not have reflected tumor PD-L1 status at the time of treatment, and that only $83 \%$ of the patients who underwent randomization had quantifiable PD-L1 expression in Brahmer2015.

However, the improved safety profile and the durability of responses to PD-1 inhibitors suggest that it might be a reasonable option for patients regardless of PD-L1 expression.

The safety profile of PD-1 inhibitors observed in this meta-analysis is consistent with that in prior prediction and was favorable compared with chemotherapy, with most 
A

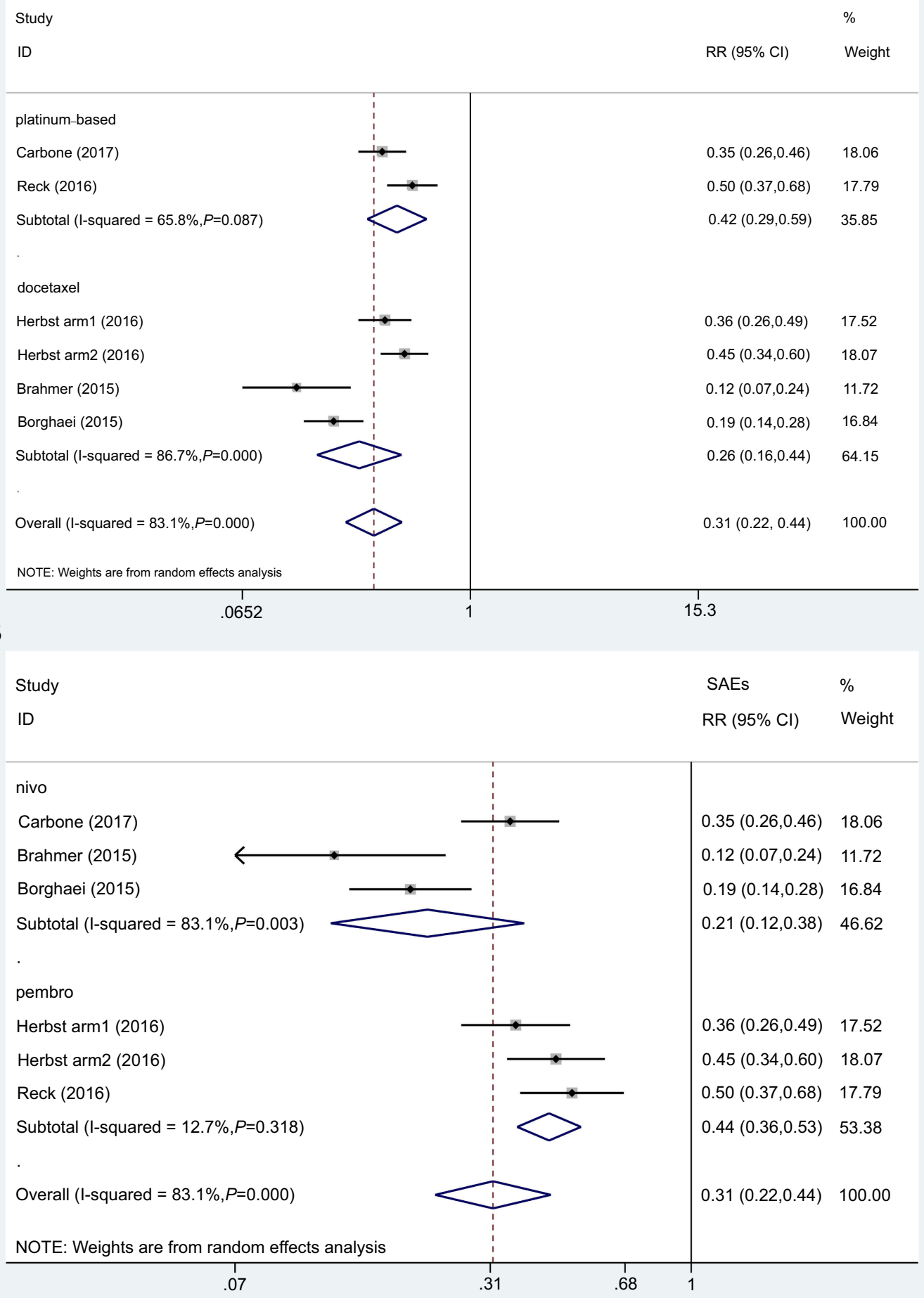

Figure 7 Subgroup risk ratio of severe adverse events. (A) Subgroup risk ratio of severe adverse events based on docetaxel and platinum duplexes. (B) Subgroup risk ratio of severe adverse events associated with PDI versus chemotherapy.

Abbreviations: SAEs, severe adverse events; RR, risk ratio.

patients having low SAEs. The frequencies of AEs includ- to discontinuation. Only a small percentage of patients in ing severe toxic events, were substantially less with PD-1 the PD-1 inhibitor group reported immune-related AEs inhibitors than with chemotherapy, similar to AEs, leading (eg, fatigue, pneumonitis, and diarrhea), which were 
infrequent and of low severity in this study; they were managed with the use of established guidelines. ${ }^{22}$ No new safety concerns were identified. Immune-mediated AEs with immunotherapies, such as PD-1 inhibitors, differed from those seen with traditional cytotoxic therapies, and particular attention should be given to rapidly evaluate and initiate the treatment. When early detection and correct management, immune-mediated side effects are basically reversible. Therefore, PD-1 inhibitors led to a statistically superior survival benefit over chemotherapy in patients with advanced NSCLC. The benefit was observed regardless of the PD-L1 expression level.

Further research is needed to identify relevant biomarkers that have sufficient sensitivity and specificity to predict which patients are most likely to benefit. ${ }^{23}$ In conclusion, PD-1 inhibitors showed a clinically meaningful survival benefit, with an improved safety profile, compared with the current standard of care in patients with advanced and previously treated NSCLC. Given that this meta-analysis included monotherapy, combined PD-1 inhibitors and chemotherapy were often used to maximize the OS in clinical practice, and the results of the combination therapy may be also better than the results of chemotherapy alone. However, considering the potential heterogeneity of combination therapy and monotherapy, the meta-analysis excluded this study because we were unable to calculate the efficacy and safety of combination therapy originating from PD-1 inhibitors or chemotherapy. Combination therapy may improve survival benefit to some extent. ${ }^{24,25}$ However, formulating evaluation criteria for the efficacy of solid tumors based on the characteristics of immunotherapy is necessary. Hence, these issues need to be further explored.

Similar to other meta-analyses, our study has several limitations. First, as we only included five RCTs in these meta-analyses, the publication bias was not assessed in this study. Second, the data were extracted from summary data rather than from the individual patients from each trial. Finally, specific biomarkers, which have sufficient sensitivity and specificity to predict which patients are most likely to benefit, could not be incorporated into analysis.

\section{Conclusion}

PD-1 inhibitors showed a clinically meaningful survival benefit and an improved safety profile in patients with previously treated NSCLC.

\section{Acknowledgments}

This research was supported by the fund for the academic backbone of the excellent young and middle-age people of Anhui Medical University (2013), the fund from The First Affiliated Hospital of Anhui Medical University for reserve talents (2014), and the fund for Excellent Top Talent Cultivation Project of Anhui Higher Education Institutions (gxyqZD2017030).

\section{Author contributions}

Ran Wang and Gengyun Sun designed and supervised the study. Yi Liu, Sijing Zhou, and Yongsheng Du drafted the manuscript, carried out the literature search, and extracted the data from the eligible studies. Li Sun, Huihui Jiang, and Binbin Zhang critically reviewed, contributed to, and approved the final manuscript. All authors contributed to data analysis, drafting and revising the article, gave final approval of the version to be published, and agree to be accountable for all aspects of the work.

\section{Disclosure}

The authors report no conflicts of interest in this work.

\section{References}

1. Puri S, Shafique M, Gray JE. Immune checkpoint inhibitors in early-stage and locally advanced non-small cell lung cancer. Curr Treat Options Oncol. 2018;19(8):39. doi:10.1007/s11864-018-0556-7

2. Schiller JH, Gandara DR, Goss GD, Vokes EE. Non-small-cell lung cancer: then and now. J Clin Res Oncol. 2013;31(8):981-983. doi: $10.1200 /$ JCO.2012.47.5772

3. Freeman GJ, Long AJ, Iwai Y, et al. Engagement of the PD-1 immunoinhibitory receptor by a novel $\mathrm{B} 7$ family member leads to negative regulation of lymphocyte activation. J Exp Med. 2000;192(7):1027-1034.

4. Keir ME, Butte MJ, Freeman GJ, Sharpe AH. PD-1 and its ligands in tolerance and immunity. Annu Rev Immunol. 2008;26:677-704. doi:10.1146/annurev.immunol.26.021607.090331

5. Butte MJ, Keir ME, Phamduy TB, Sharpe AH, Freeman GJ. Programmed death-1 ligand 1 interacts specifically with the B7-1 costimulatory molecule to inhibit $\mathrm{T}$ cell responses. Immunity. 2007;27(1):111-122. doi:10.1016/j.immuni.2007.05.016

6. Cyriac G, Gandhi L. Emerging biomarkers for immune checkpoint inhibition in lung cancer. Semin Cancer Biol. 2018;52:269-277. doi:10.1016/j.semcancer.2018.05.006

7. Wang C, Thudium KB, Han M, et al. In vitro characterization of the anti-PD-1 antibody nivolumab, BMS-936558, and in vivo toxicology in non-human primates. Cancer Immunol Res. 2014;2(9):846-856. doi:10.1158/2326-6066.CIR-14-0040

8. Cheng X, Veverka V, Radhakrishnan A, et al. Structure and interactions of the human programmed cell death 1 receptor. J Biol Chem. 2013;288(17):11771-11785. doi:10.1074/jbc.M112.448126

9. Hanahan D, Weinberg RA. Hallmarks of cancer: the next generation. Cell. 2011;144(5):646-674. doi:10.1016/j.cell.2011.02.013 
10. Pardoll DM. The blockade of immune checkpoints in cancer immunotherapy. Nat Rev Cancer. 2012;12(4):252-264. doi:10.1038/ nrc3239

11. Feld E, Horn L. Targeting PD-L1 for non-small-cell lung cancer. Immunotherapy. 2016;8(6):747-758. doi:10.2217/imt-2016-0012

12. Higgins JP, Altman DG, Gotzsche PC, et al. The Cochrane Collaboration's tool for assessing risk of bias in randomised trials. Bmj. 2011;343:d5928. doi:10.1136/bmj.d5928

13. Horn L, Spigel DR, Vokes EE, et al. Nivolumab versus docetaxel in previously treated patients with advanced non-small-cell lung cancer: two-year outcomes from two randomized, open-label, Phase III trials (CheckMate 017 and CheckMate 057). J Clin Res Oncol. 2017;35 (35):3924-+. doi:10.1200/JCO.2017.74.3062

14. Carbone DP, Reck M, Paz-Ares L, et al. First-line nivolumab in stage IV or recurrent non-small-cell lung cancer. $N$ Engl J Med. 2017;376 (25):2415-2426. doi:10.1056/NEJMoa1613493

15. Reck M, Rodriguez-Abreu D, Robinson AG, et al. Pembrolizumab versus chemotherapy for PD-L1-positive non-small-cell lung cancer. $N$ Engl J Med. 2016;375(19):1823-1833. doi:10.1056/NEJMoa1606774

16. Herbst RS, Baas P, Kim DW, et al. Pembrolizumab versus docetaxel for previously treated, PD-L1-positive, advanced non-small-cell lung cancer (KEYNOTE-010): a randomised controlled trial. Lancet (London, England). 2016;387(10027):1540-1550. doi:10.1016/ S0140-6736(15)01281-7

17. Borghaei H, Paz-Ares L, Horn L, et al. Nivolumab versus docetaxel in advanced nonsquamous non-small-cell lung cancer. $N$ Engl J Med. 2015;373(17):1627-1639. doi:10.1056/NEJMoa1507643
18. Brahmer J, Reckamp KL, Baas P, et al. Nivolumab versus docetaxel in advanced squamous-cell non-small-cell lung cancer. $N$ Engl J Med. 2015;373(2):123-135. doi:10.1056/NEJMoa1504627

19. Champiat S, Ferte C, Lebel-Binay S, Eggermont A, Soria JC. Exomics and immunogenics: bridging mutational load and immune checkpoints efficacy. Oncoimmunology. 2014;3(1):e27817. doi:10.4161/onci.27817

20. Rizvi NA, Hellmann MD, Snyder A, et al. Cancer immunology. Mutational landscape determines sensitivity to PD-1 blockade in non-small cell lung cancer. Science. 2015;348(6230):124-128. doi:10.1126/science.aaa1348

21. Attili I, Passaro A, Pavan A, Conte P, De Marinis F, Bonanno L. Combination immunotherapy strategies in advanced non-small cell lung cancer (NSCLC): does biological rationale meet clinical needs? Crit Rev Oncol Hematol. 2017;119:30-39. doi:10.1016/j.critrevonc.2017.09.007

22. Oppel-Heuchel H, Grimm MO. [Therapy monitoring and management of adverse events in PD-1/PD-L1 immune checkpoint inhibition]. Der Urologe Ausg A. 2016;55(5):677-690. doi:10.1007/ s00120-016-0109-2

23. Vanneman M, Dranoff G. Combining immunotherapy and targeted therapies in cancer treatment. Nat Rev Cancer. 2012;12(4):237-251. doi: $10.1038 / \mathrm{nrc} 3237$

24. Hellmann MD, Ciuleanu TE, Pluzanski A, et al. Nivolumab plus ipilimumab in lung cancer with a high tumor mutational burden. $N$ Engl J Med. 2018;378(22):2093-2104. doi:10.1056/NEJMoa1801946

25. Yoneda K, Imanishi N, Ichiki Y, Tanaka F. Immune checkpoint inhibitors (ICIs) in non-small cell lung cancer (NSCLC). J Uoeh. 2018;40(2):173-189. doi:10.7888/juoeh.40.173

\section{Publish your work in this journal}

Cancer Management and Research is an international, peer-reviewed open access journal focusing on cancer research and the optimal use of preventative and integrated treatment interventions to achieve improved outcomes, enhanced survival and quality of life for the cancer patient.
The manuscript management system is completely online and includes a very quick and fair peer-review system, which is all easy to use. Visit http://www.dovepress.com/testimonials.php to read real quotes from published authors. 